\title{
Relaxed Ziegler-Nichols Closed Loop Tuning of PI Controllers
}

\author{
Finn Haugen ${ }^{1}$ Bernt Lie $^{1}$
}

Telemark University College, Kjolnes ring 56, 3918 Porsgrunn, Norway. E-mail contact: \{ finn. haugen, bernt.lie\} @hit.no

\begin{abstract}
A modification of the PI setting of the Ziegler-Nichols closed loop tuning method is proposed. The modification is based on a combination of the Skogestad SIMC tuning formulas for "integrator plus time-delay" processes with the Ziegler-Nichols tuning formulas assuming that the process is modeled as an "integrator plus time-delay" process. The resulting PI settings provide improved stability margins compared with those obtained with the original Ziegler-Nichols PI settings. Compared with the well-known TyreusLuyben PI settings, the proposed PI settings give improved disturbance compensation. For processes with zero or a negligible time-delay, but with some lags in the form of time-constants, tuning based on ultimate gain and ultimate period may give poor results. Successful PI settings for such processes are proposed.
\end{abstract}

Keywords: PI controller, tuning, open loop, closed loop, Ziegler-Nichols, Tyreus-Luyben, Skogestad, relay-tuning, performance, stability, robustness.

\section{Introduction}

The PI (proportional plus integral) controller is probably the most frequently used controller function in practical applications. The PI controller stems from a PID controller with the D-term (derivative) deactived to reduce the propagation of amplified random measurement noise via the controller, thereby limiting variations in the control signal due to noise.

Ziegler and Nichols (1942) presented two, now famous, methods for tuning P, PI, and PID controllers: The closed loop, or ultimate gain, method, and the open loop, or process reaction curve, method. In the present paper, focus is on closed loop tuning of PI controllers.

The PI settings with the Ziegler and Nichols closed loop method are:

$$
\begin{gathered}
K_{c}=0.45 K_{c_{u}} \\
T_{i}=\frac{P_{u}}{1.2}
\end{gathered}
$$

where $K_{c_{u}}$ is the ultimate gain, and $P_{u}$ is the ultimate period to be found by the user. A practical, experimental way to find $K_{c_{u}}$ and $P_{u}$ is using relay oscillations, Åstrøm and Hägglund (1995), cf. Appendix A.

It is well-known that Ziegler and Nichols closed loop PI tuning in many cases give relatively fast process disturbance compensation, but unfortunately poor stability margins, seen as poorly damped oscillatory responses. This is demonstrated in several examples in Section 3. Tyreus and Luyben (1992) proposed a now well-known modification of the Ziegler-Nichols PI settings which typically give improved control system stability:

$$
\begin{gathered}
K_{c}=0.31 K_{c_{u}} \\
T_{i}=2.2 P_{u}
\end{gathered}
$$

In the present paper, another modification of the Ziegler-Nichols PI settings is proposed to provide acceptable stability margins and improved disturbance compensation compared to the Tyreus and Luyben settings. The proposed tuning rules, here denoted the Relaxed Ziegler-Nichols (R-ZN) PI settings, are based 
on the open loop tuning rules in the SIMC method (Simple Internal Model Control) by Skogestad (2004) applied to an "integrator plus time-delay" process estimated from the ultimate gain and the ultimate period, Yu (1999).

The outline of this paper is as follows: In Section 2, the R-ZN PI settings are derived. In Section 3, the original Ziegler-Nichols (ZN) PI settings, the Relaxed Ziegler-Nichols PI settings, and the Tyreus-Luyben (TL) PI settings are applied to two simulation cases and to a practical temperature control system of an air heater. In Section 4 an adjustable parameter of the R-ZN method is used to tune processes without timedelay, but with lags. Section 5 contains a discussion, and conclusions are given in Section 6.

Appendix A reviews the relay experiment of finding the ultimate gain and the ultimate period from both sinusoidal and triangular oscillations. Appendix B presents a modification of the Skogestad PI settings for improved disturbance compensation, used in the derivation of the proposed PI controller setting. Appendix $\mathrm{C}$ shows abbreviations and nomenclature.

In this paper, the same symbol (letter) will be used for variables in time-domain as in the Laplace domain. This simplifies the notation. It is assumed that the meaning of the symbol is clear from the context.

MATLAB and SIMULINK (MathWorks, Inc.) are used for numerical computations and simulations. LabVIEW (National Instruments, Inc.) is used to implement the temperature control system for the real air heater.

\section{Relaxed Ziegler-Nichols PI tuning}

\subsection{Derivation of the tuning formulas}

The following PI controller function is assumed:

$$
u(t)=u_{\operatorname{man}}+K_{c} e(t)+\frac{K_{c}}{T_{i}} \int_{0}^{t} e(\tau) d \tau
$$

Skogestad (2004) has provided PI settings for a number of different types of process dynamics, among which are "integrator plus time-delay" and "timeconstant plus time-delay". Assuming that Skogestad's rule-of-thumb about setting the user-specified closed loop time-constant, $T_{c}$, equal to the process time-delay, $\tau$, his PI settings for these two process types are actually identical as long as the relation between the timeconstant of the "time-constant plus time-delay" process and the time-delay satisfies

$$
T \geq 8 \tau
$$

In the following, it is assumed that eq. (6) is satisfied for the process to be controlled. Thus, an "integrator plus time-delay" process is assumed, with the following transfer function:

$$
\frac{\Delta y(s)}{\Delta u(s)}=H_{p}(s)=\frac{K_{i_{p}}}{s} e^{-\tau s}
$$

The Skogestad PI settings for this process are:

$$
\begin{gathered}
K_{c}=\frac{1}{K_{i_{p}}\left(T_{c}+\tau\right)} \\
T_{i}=c_{s}\left(T_{c}+\tau\right)
\end{gathered}
$$

The parameter $c_{s}$ is introduced here. The original PI settings in Skogestad (2004) correspond to $c_{s}=4$ in eq. (9). For "integrator plus time-delay" processes with an "input" process disturbance, the disturbance compensation appears as unnecessarily slow with $c_{s}=$ 4. To obtain a faster disturbance compensation while retaining acceptable stability margins, a value of $c_{s}$ smaller than 4 can be used. It is found that values around 2 are proper values. Thus, $c_{s}=2$ is proposed. The implications of various values of $c_{s}$ are investigated in Appendix B.

The user must select a proper value of $T_{c}$ in eqs. (8) and (9). Skogestad provides the following rule-ofthumb:

$$
T_{c}=\tau
$$

With $c_{s}=2$ and the rule-of-thumb eq. (10), eqs. (8) and (9) become

$$
\begin{gathered}
K_{c}=\frac{1}{2 K_{i_{p}} \tau} \\
T_{i}=4 \tau
\end{gathered}
$$

which may be denoted the modified Skogestad PI settings for "integrator plus time-delay" processes.

The Skogestad PI settings, also with $c_{s}=2$, typically yield acceptable stability of the control system, while Ziegler and Nichols PI settings often give poor stability, with oscillatory responses (as demonstrated in several applications in Section 3). The PI settings, eqs. (11) and (12), will now be exploited to relax the original ZN PI settings, eqs. (1)-(2).

For an "integrator plus time-delay" process, $K_{i_{p}}$ and $\tau$ can be estimated from $K_{c_{u}}$ and $P_{u}$ as follows, Yu (1999), DiRuscio (2010):

$$
\begin{aligned}
K_{i_{p}} & =\frac{2 \pi}{K_{c_{u}} P_{u}} \\
\tau & =\frac{P_{u}}{4}
\end{aligned}
$$

As pointed out in Seborg et al. (2004), process parameters $K_{i_{p}}$ and $\tau$ can be used in any model-based controller tuning method. Here, the (modified) Skogestad 
PI settings, eqs. (11) and (12), are used. Inserting eqs. (13) and (14) into eqs. (11) and (12) gives

$$
\frac{K_{c}=\frac{K_{c_{u}}}{\pi}=0.32 K_{c_{u}}}{\underline{T_{i}=P_{u}}}
$$

which will be referred to as the (default) Relaxed Ziegler-Nichols (R-ZN) PI settings.

\section{Comparing with ZN and TL}

Compared with the (original) ZN PI settings, eqs. (1)(2), the gain is smaller and the integral time is somewhat larger in the R-ZN PI settings, indicating improved stability.

Compared with the TL PI settings, eqs. (3)-(4), the R-ZN gain is almost the same, while the R-ZN integral time is smaller, indicating faster integral action, i.e. the control error is brought faster to zero, however, somewhat reduced stability can be expected.

\section{Enhanced relaxation}

Above, the closed loop time-constant is set equal to the (estimated) process time-delay, cf. eq. (10). Particularly in applications where the process has zero or negligible time-delay but some lag, the default R-ZN PI settings may result in poor stability (and the ZN settings may even give instability). Acceptable stability can be obtained with enhanced relaxation of the PI settings. To this end, we propose

$$
\underline{T_{c}=k_{r} \tau}
$$

where $k_{r} \geq 1$ is a relaxation parameter to be set by the user. The default PI settings, eqs. (6) and (6), are obtained with $k_{r}=1$. Enchanced relaxation of the PI settings is obtained with $k_{r}>1$. Using eq. (17) in eqs. (8) and (9), and setting $c_{s}=2$ in (9), give

$$
K_{c}=\frac{2}{\pi\left(k_{r}+1\right)} K_{c_{u}}
$$

and

$$
\underline{T_{i}=\frac{k_{r}+1}{2} P_{u}}
$$

The usefulness of enhanced R-ZN tuning is demonstrated in Section 4.

One question may arise: Why not just apply original ZN settings and adjust $K_{c}$ and $T_{i}$ directly? While this is of course an option, we think that it better to use a meaningful single parameter, $k_{r}$, to obtain the PI settings. The benefit of reducing the number of controller parameters to adjust from two to one is actually substantial. Skogestad's tuning method is an excellent example of this: From the user's perspective, adjusting $T_{c}$, which has a meaningful interpretation, to obtain the PI settings is a much simpler task than adjusting $K_{c}$ and $T_{i}$ directly.

\subsection{Some derived results}

\section{Estimation of control system response-time}

The control system response-time, $T_{r}$, can be estimated from the ultimate period, $P_{u}$, as explained in the following. The typical setting of $k_{r}=1$ is here assumed. Then the PI settings are eqs. (6) and (6). Assume that the setpoint is changed as a step. Then the response in the process output reaches $63 \%$ of its final value at time (approximately)

$$
T_{r} \approx \tau+T_{c}=\frac{P_{u}}{4}+\frac{P_{u}}{4}=\frac{P_{u}}{2}
$$

$T_{r}$ is here the $63 \%$ rise-time, or response-time, of the control system. As an example of eq. (20), see Figure 9 where the response in air heater temperature due to a setpoint step is plotted. In that example, $P_{u}=15 \mathrm{~s}$, giving $T_{r} \approx P_{u} / 2=7.5 \mathrm{~s}$, which is in good accordance with the plotted response in Figure 9.

\section{Retuning the PI controller}

Equations (18) and (19) can be used to retune a PI controller safely. Note that the factor $\left(k_{r}+1\right)$ appears in the denominator of eq. (18) and in the numerator of eq. (19). For example, assume that it is desired to decrease the present value of $K_{c}$ by a factor of 2 (to obtain a smoother control signal). This gain reduction should be acccompanied by an increase of $T_{i}$ by a factor 2. (This inversely proportional adjustment also follows directly from Skogestad's formulas, eqs. (8) and (9).)

\section{Applications}

\subsection{Overview}

In the following subsections, PI settings with the (original) Ziegler-Nichols closed loop method, the R-ZN closed loop method, and the TL method are applied to the following three cases:

- A simulated control system for an "integrator with time-delay" process (Section 3.3).

- A simulated control system for a "time-constant with time-delay" process (Section 3.4).

- A practical temperature control system for a laboratory air heater (Section 3.5). The process dynamics is roughly "time-constant with timedelay". 
The PI settings will be compared using quantitive measures of performance and robustness defined in Section 3.2.

For easy reference, the various PI settings formulas are summarized in Table 1 . In the examples, $K_{c_{u}}$ and $P_{u}$ are found from the method of relay oscillations described in Appendix A.

Table 1: PI settings formulas.

\begin{tabular}{c|ccc} 
& $\mathbf{Z N}$ & $\mathbf{R}-\mathbf{Z N}$ & $\mathbf{T L}$ \\
\hline$K_{c}$ & $0.45 K_{c_{u}}$ & $0.32 K_{c_{u}}$ & $0.31 K_{c_{u}}$ \\
$T_{i}$ & $\frac{P_{u}}{1.2}$ & $P_{u}$ & $2.2 P_{u}$
\end{tabular}

\subsection{Measures of performance and robustness}

The measures used in this paper for comparing the various methods of PI controller tuning can be grouped into performance and robustness measures described in the detail in the following.

\subsubsection{Performance}

\section{IAE at setpoint change}

In the tests the setpoint is changed as a step. The setpoint tracking is measured with the IAE (Integral of Absolute Error) index calculated over a proper time interval as

$$
\mathrm{IAE}_{s}=\int_{t_{i}}^{t_{f}}|e| d t
$$

where $e$ is the control error, $t_{i}$ is the initial time, selected as the time of the step change, and $t_{f}$ is a proper final time. A reduced $\mathrm{IAE}_{s}$ value indicates improved setpoint tracking.

\section{IAE at process disturbance change}

In the tests a process disturbance is changed as a step. The disturbance compensation is measured with

$$
\mathrm{IAE}_{d}=\int_{t_{i}}^{t_{f}}|e| d t
$$

A reduced $\mathrm{IAE}_{d}$ value indicates improved disturbance compensation.

\section{Response time}

The response time, $T_{r}[\mathrm{~s}]$, is here defined as the inverse of the bandwidth defined as the amplitude crossover frequency, $\omega_{c}[\mathrm{rad} / \mathrm{s}]$ :

$$
T_{r}=\frac{1}{\omega_{c}}
$$

$T_{r}$ indicates the speed of the response of the control system due to a setpoint step change. $T_{r}$ is approximately the time-constant of the control system. $\omega_{c}$ is equal to the phase crossover frequency, $\omega_{180_{d}}$, of the loop brought to marginal stability by a reduction of the phase of the loop while the amplitude is retained, as by an increase of the loop time-delay:

$$
T_{r}=\frac{1}{\omega_{180_{d}}}=\frac{P_{u}}{2 \pi}
$$

where $P_{u}[\mathrm{~s}]$ is the (ultimate) period of the oscillations at marginal stability.

\section{Setpoint tracking versus disturbance compensation}

For systems where the setpoint is constant, which is the case in many practical process control systems, it can be claimed that good disturbance compensation is more important than good setpoint tracking. In the examples presented in the following sections, disturbance compensation is emphasized.

\subsubsection{Stability robustness (stability margins)}

\section{Gain margin, GM}

For the cases based on simulations GM is calculated from the loop transfer function, $H_{L}(s)$, using the margin function in MATLAB. $H_{L}(s)$ is

$$
H_{L}(s)=H_{c}(s) H_{p}(s)
$$

where $H_{c}(s)$ is the controller transfer function, and $H_{p}(s)$ is the process transfer function.

For the practical case (air heater) an adjustable gain, $\Delta K$, is inserted into the loop (between the controller and the process), see Figure 1 . Initially, $\Delta K=1$. The

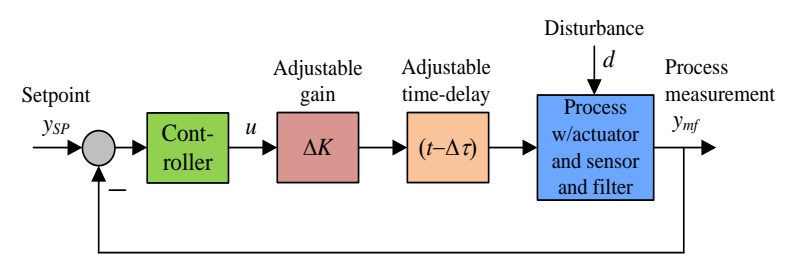

Figure 1: An adjustable gain and time-delay are inserted into the loop to find the stability margins (gain margin and phase margin) experimentally.

(ultimate) value $\Delta K_{u}$ that brings the control system to the stability limit so that the responses are sustained oscillations, is found experimentally (by trials). The gain margin is then

$$
\mathrm{GM}=\Delta K_{u}
$$




\section{Phase margin, PM}

For the cases based on simulations PM is calculated from the loop transfer function using the margin function in MATLAB.

For the practical case (air heater) an adjustable timedelay, $\Delta \tau[\mathrm{s}]$, is inserted into the loop (between the controller and the process), see Figure 1. Initially, $\Delta \tau=0$. For each of the tuning methods, the value $\Delta \tau_{u}$ that brings the control system to the stability limit, i.e. causing sustained oscillations, is found experimentally. The period, $P_{u}[\mathrm{~s}]$, of the oscillations is measured. The corresponding phase margin is

$$
\mathrm{PM}[\mathrm{deg}]=360 \frac{\Delta \tau_{u}}{P_{u}}
$$

Equation (27) is derived in Haugen (2012) (Appendix 1).

\section{Proper values of GM and PM}

Seborg et al. (2004) propose the following ranges for proper values of the stability margins:

$$
1.7=4.6 \mathrm{~dB} \leq \mathrm{GM} \leq 4.0=12.0 \mathrm{~dB}
$$

and

$$
30^{\circ} \leq \mathrm{PM} \leq 45^{\circ}
$$

Since poor control system stability must be avoided, the lower limits of GM and PM can be regarded as critical, while the upper limits are not.

\subsection{Application: Simulated "integrator plus time-delay" process}

\subsubsection{Process description}

The process to be controlled is an "integrator plus time-delay" process:

$$
\dot{y}(t)=K_{i_{p}} u(t-\tau)+K_{d} d(t)
$$

which has transfer function as in eq. (7). The process parameter values are: $K_{i_{p}}=1 \mathrm{~s}^{-1}, K_{d}=1, \tau=1 \mathrm{~s}$.

\subsubsection{PI controller tuning from relay oscillations}

$K_{c_{u}}$ and $P_{u}$ are found from relay oscillations. Figure 2 shows plots of the sustained oscillations during the relay tuning, cf. Appendix A. From the plots, $A_{\text {tri }}=1.0$. The square wave in the control signal has amplitude $A_{\text {sq }}=1$.

Equation (47) in Appendix A gives

$$
K_{c_{u}}=\frac{\pi A_{s q}}{2 A_{t r i}}=\frac{\pi \cdot 1}{2 \cdot 1}=1.57
$$
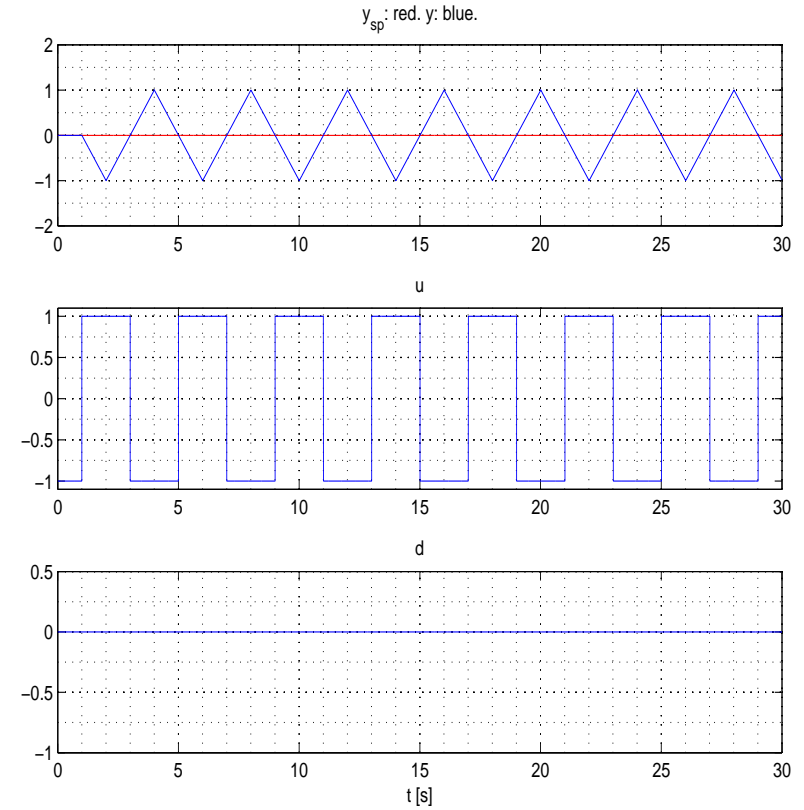

Figure 2: Responses during relay tuning

It is interesting that the ultimate gain using a $\mathrm{P}$ controller also gives $K_{c_{u}}=1.57$. Hence, the Fourier-series approximations used to derive eq. (47) give a very precise result in this case.

Furthermore, from the plots,

$$
P_{u}=4.0 \mathrm{~s}
$$

Various PI settings are calculated from the above values of $K_{c_{u}}$ and $P_{u}$ using the formulas in Table 1. The PI settings are shown in Table 2.

\subsubsection{Performance and stability robustness of the control system}

Figure 3 shows responses in the process output variable $(y)$ and the controller output $(u)$ with a step change of the temperature setpoint $\left(y_{s p}\right)$ and a step change of the disturbance $(d)$ for the three different PI settings shown in Table 2.

GM, PM and $T_{r}$ are calculated from the model. $\mathrm{IAE}_{s}$ is calculated time-series over the interval $t=$ $[2 \mathrm{~s}, 40 \mathrm{~s}] . \mathrm{IAE}_{d}$ is calculated over $t=[40 \mathrm{~s}, 80 \mathrm{~s}]$. Table 2 summarizes the performance and robustness measures.

Below are a number of observations made in Table 2 (the abbreviations are as in Table 2):

- Setpoint tracking:

$\mathrm{IAE}_{s}: \mathrm{ZN}$ and TL are the best, and almost equal, but ZN suffers from large overshoot. 

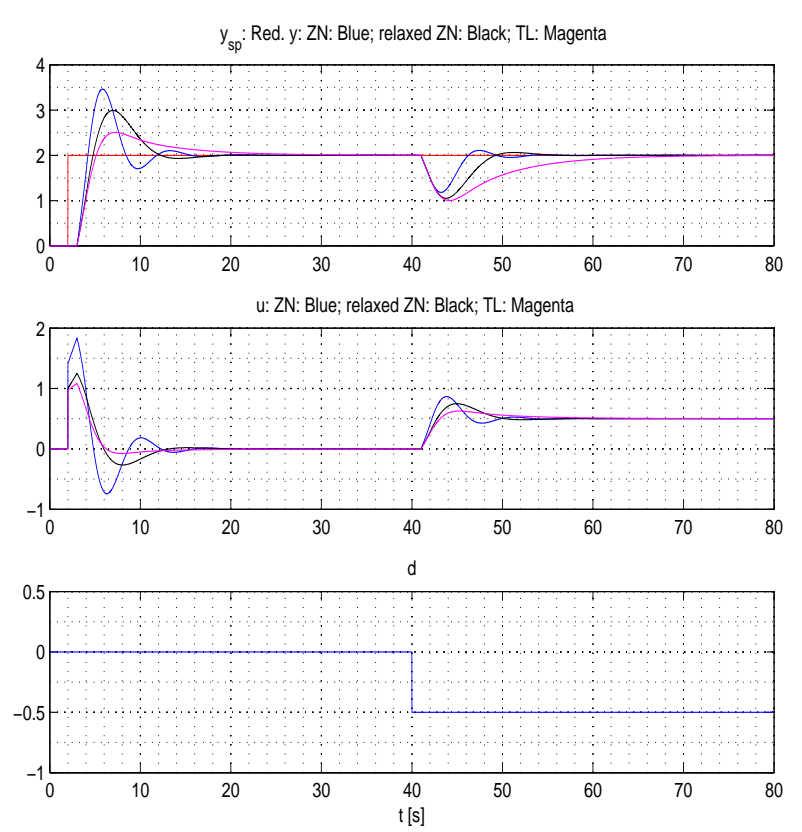

Figure 3: Responses with various PI settings.

Table 2: Controller settings and performance and robustness measures for simulated control system for "integrator plus time-delay" process with different PI settings.

\begin{tabular}{c|ccc} 
& ZN & R-ZN & TL \\
\hline$K_{c}$ & 0.71 & 0.50 & 0.49 \\
$T_{i}[\mathrm{~s}]$ & 3.3 & 4.0 & 8.8 \\
$\mathrm{IAE}_{s}$ & 7.9 & 8.1 & 8.0 \\
$\mathrm{IAE}_{d}$ & 2.8 & 4.5 & 9.0 \\
$\mathrm{GM}$ & 1.9 & 2.7 & 3.1 \\
$\mathrm{GM}[\mathrm{dB}]$ & 5.4 & 8.8 & 9.7 \\
$\mathrm{PM}[\mathrm{deg}]$ & 24.9 & 34.1 & 48.6 \\
$T_{r}[\mathrm{~s}]$ & $1.3 \mathrm{~s}$ & 1.8 & 2.0
\end{tabular}

$T_{r}: \mathrm{ZN}$ is the best, while R-ZN and TL do not differ much.

- Disturbance compensation:

$\mathrm{IAE}_{d}: \mathrm{ZN}$ is clearly best. R-ZN is in turn clearly better than TL as the R-ZN has a value which is $50 \%$ of the value of TL.

- Stability robustness (margins):

GM: ZN is poor, and actually below the lower limit in ineq. (28). R-ZN and TL do not differ much and have acceptable values.

PM: Again ZN is poor, and below the lower limit in ineq. (29). R-ZN gives a somewhat small, but acceptable, value. TL gives large value, possibly unnecessarily large as it is larger than the higher limit in ineq. (29).

The low stability margins with $\mathrm{ZN}$ are apparent in the oscillatory responses with the ZN settings, see Figure 3.

\section{Comments and conclusions}

The Ziegler-Nichols PI settings give poor control loop stability margins. The TL and the R-ZN settings give acceptable stability margins. With emphasis on disturbance compensation rather than setpoint tracking, the R-ZN settings are better than the TL settings.

\subsection{Application: Simulated "time-constant plus time-delay" process}

\subsubsection{Process description}

The process to be controlled is a "time-constant plus time-delay" process (assuming the time-delay is at the input-side):

$$
T \dot{y}(t)=-y(t)+K u(t-\tau)+K_{d} d(t)
$$

The process parameter values are: $K=8, K_{d}=8, \tau=$ $1 \mathrm{~s}$.

The time-constant being 8 times the time-delay makes the Skogestad PI settings for a "time-constant plus time-delay" process become identical with the settings for an "integrator plus time-delay" process. Therefore, the condition for using Skogestad tuning for "integrator plus time-delay" processes, ineq. (6), is satisfied.

\subsubsection{PI controller tuning from relay oscillations}

The ultimate gain and the ultimate period are found from relay oscillations. Figure 4 shows plots of the sustained oscillations during the relay tuning. The response in $y$ are approximately triangular, so eq. (47) is used to calculate $K_{c_{u}}$. From Figure $4, A_{\text {tri }}=0.94$, $A_{\mathrm{sq}}=1$. Equation (47) gives

$$
K_{c_{u}}=\frac{\pi A_{\mathrm{sq}}}{2 A_{\mathrm{tri}}}=\frac{\pi \cdot 0.94}{2 \cdot 1}=1.48
$$

The ultimate gain using a $\mathrm{P}$ controller gives $K_{c_{u}}=1.65$ which differs somewhat from 1.48. Still, $K_{c_{u}}=1.48$ is used to stick to relay tuning, and using 1.48 rather than 1.65 is safe (conservative) regarding control loop stability.

Furthermore, from Figure 4,

$$
P_{u}=3.78 \mathrm{~s}
$$



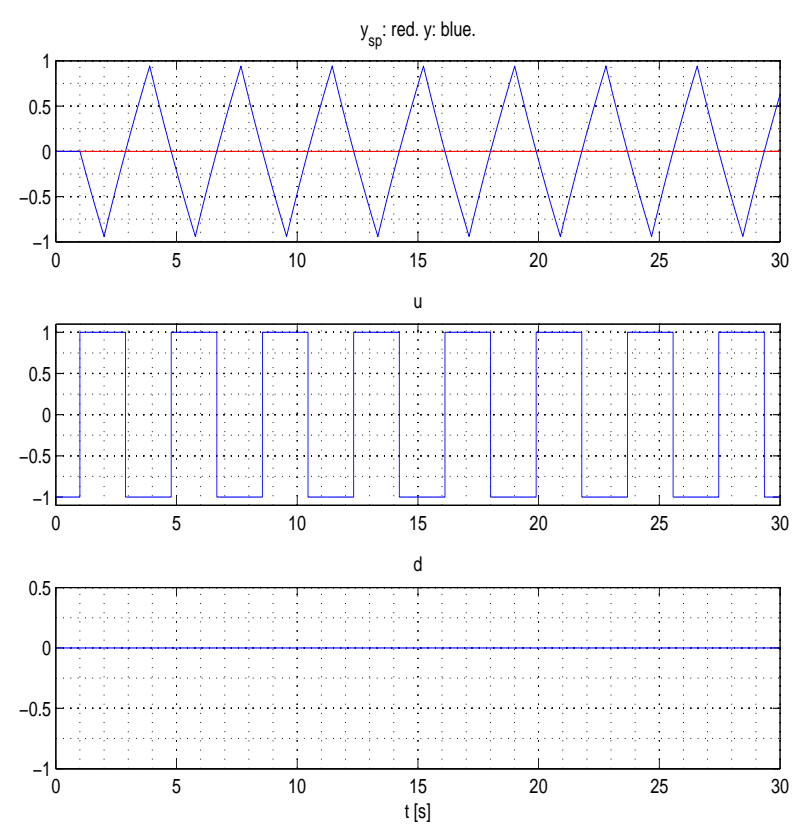

Figure 4: Responses during relay tuning

Various PI settings are calculated from the above values of $K_{c_{u}}$ and $P_{u}$ using the formulas in Table 1. The PI settings are shown in Table 2.

\subsubsection{Performance and stability robustness of the control system}

Figure 5 shows responses in the process output variable $(y)$ and the controller output $(u)$ with a step change of the temperature setpoint $\left(y_{s p}\right)$ and a step change of the disturbance $(d)$ for the three different PI settings shown in Table 3.

GM, PM and $T_{r}$ are calculated from the model. $\mathrm{IAE}_{s}$ is calculated over the interval $t=[2 \mathrm{~s}, 40 \mathrm{~s}] . \mathrm{IAE}_{d}$ is calculated over $t=[40 \mathrm{~s}, 80 \mathrm{~s}]$. Table 3 summarizes the performance and robustness measures.

Table 3: Controller settings and performance and robustness measures for simulated control system for "time-constant plus time-delay" process with different PI settings.

\begin{tabular}{c|ccc} 
& ZN & R-ZN & TL \\
\hline$K_{c}$ & 0.75 & 0.53 & 0.52 \\
$T_{i}[\mathrm{~s}]$ & 3.2 & 3.8 & 8.3 \\
$\mathrm{IAE}_{s}$ & 6.1 & 5.8 & 4.3 \\
$\mathrm{IAE}_{d}$ & 2.1 & 3.6 & 7.9 \\
$\mathrm{GM}$ & 1.7 & 2.6 & 3.0 \\
$\mathrm{GM}[\mathrm{dB}]$ & 4.8 & 8.1 & 9.7 \\
$\mathrm{PM}[\mathrm{deg}]$ & 22.3 & 32.2 & 60.1 \\
$T_{r}[\mathrm{~s}]$ & 1.2 & 1.7 & 1.9
\end{tabular}
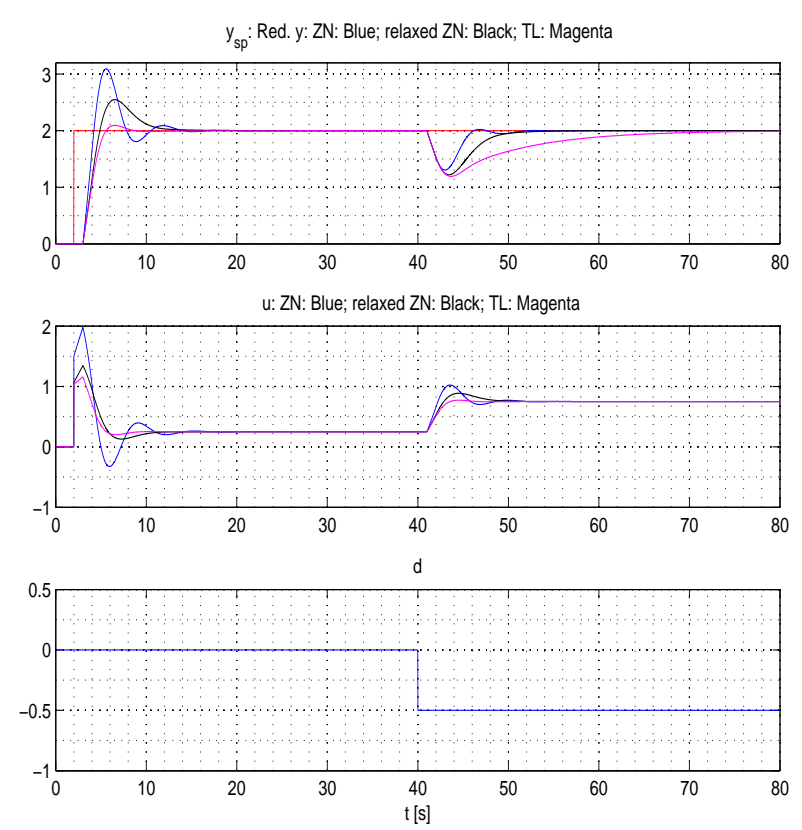

Figure 5: Responses with various PI settings.

Below are a number of observations made in Table 2 (the abbreviations are as in Table 2):

- Setpoint tracking:

$\mathrm{IAE}_{s}: \mathrm{TL}$ is best.

$T_{r}: \mathrm{ZN}$ is best, while R-ZN and TL do not differ much.

- Disturbance compensation:

$\mathrm{IAE}_{d}: \mathrm{ZN}$ is clearly best. R-ZN is in turn clearly better than TL. R-ZN has a value which is $45 \%$ of the value of TL.

- Stability robustness (margins):

GM: Strictly, all settings give acceptable values, but $\mathrm{ZN}$ is on the lower limit.

PM: $\mathrm{ZN}$ is poor, and below the lower limit in ineq. (29). R-ZN gives a somewhat small, but acceptable, value. TL gives a large value, possibly unnecessarily large as it is larger than the higher limit in ineq. (29).

\section{Comments and conclusions}

The Ziegler-Nichols PI settings give poor control loop stability as the PM is too small. The rest of the comments are identical with those for the "integrator plus time-delay" case in Section 3.3: The TL and the R-ZN settings give acceptable stability margins. With emphasis on disturbance compensation rather than setpoint tracking, the R-ZN settings are better than the TL settings. 


\subsection{Application: Practical temperature control system}

\subsubsection{Process description}

Figure 6 shows an air heater laboratory station. The

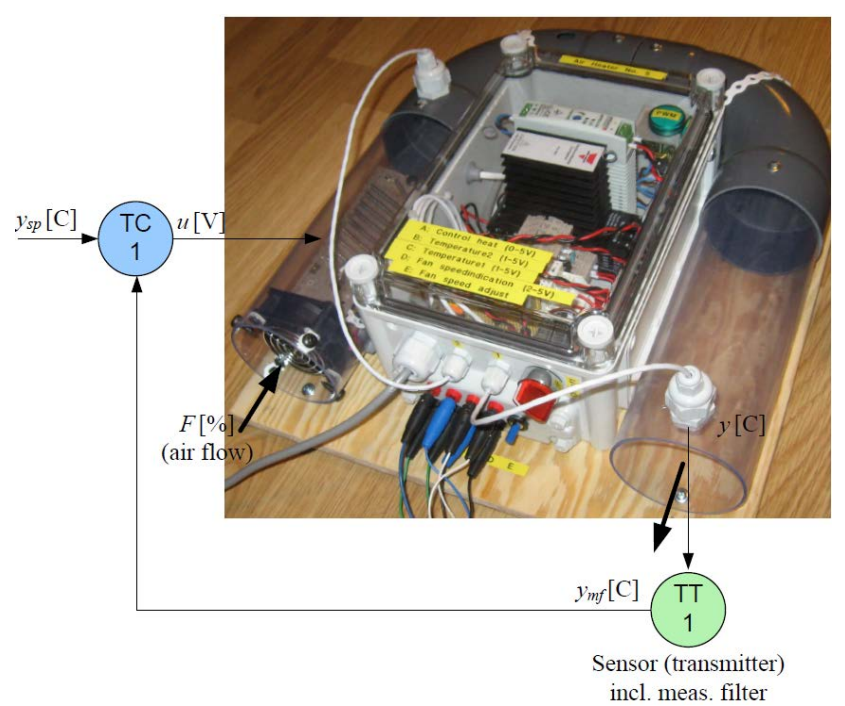

Figure 6: Temperature control system for an air heater (laboratory rig)

temperature of the air outlet is controlled by adjusting the control signal (voltage) to the heater. The temperature is measured with a Pt100 element. A measurement filter with time-constant $0.5 \mathrm{~s}$ is used to attenuate measurement noise. The National Instruments USB6008 is used as analog I/O device. The control system is implemented in LabVIEW (National Instruments) running on a PC. The fan rotational speed, and the air flow, can be adjusted manually with a potentiometer. Changes of the air flow comprises a process disturbance giving an impact on the temperature. The measured voltage drop across the potensiometer is represented by the variable $F$ in percent. Thus, $F$ represents the air flow disturbance. ${ }^{1}$

The nominal operating point of the system is temperature at $35{ }^{\circ} \mathrm{C}$ and air flow $F=50 \%$.

Figure 7 shows the open loop, or process, step response in the filtered temperature, $y_{m f}$, due to a step in the heater control signal, $u$. The response indicates that the process dynamics is roughly "time-constant with time-delay", with time-constant $\approx 37$ s and timedelay $\approx 3$ s which is about $8 \%$ of the time-constant.

\footnotetext{
1 Additional information about the air heater is available at Haugen (2013).
}
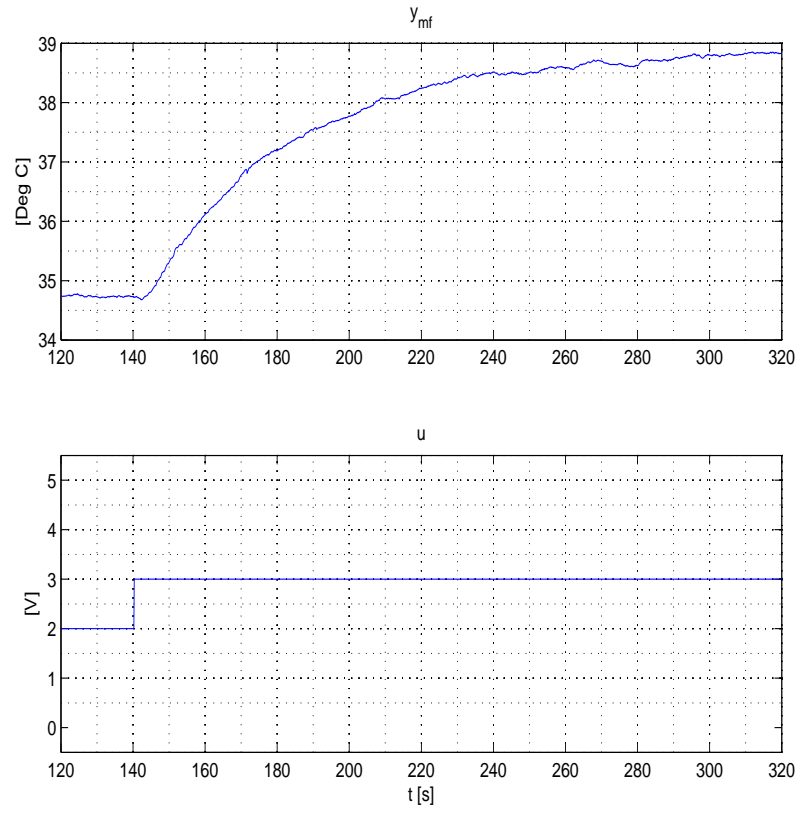

Figure 7: Open loop step response in filtered temperature, $y_{m f}$, due to a step in the heater control signal, $u$.

\subsubsection{PI controller tuning from relay oscillations}

$K_{c_{u}}$ and $P_{u}$ are found from relay oscillations. Figure 8 shows plots of the sustained oscillations during the relay tuning. The oscillations in temperature (process measurement) looks more sinusoidal than triangular. Therefore, $K_{c_{u}}$ is calculated using eq. (45).

From Figure $8, A_{\text {sin }}=0.75{ }^{\circ} \mathrm{C}$ and $A_{\mathrm{sq}}=2.5 \mathrm{~V}$. Equation (45) gives

$$
K_{c_{u}}=\frac{4 A_{\mathrm{sq}}}{\pi A_{\mathrm{sin}}}=\frac{4 \cdot 2.5 \mathrm{~V}}{\pi \cdot 0.75^{\circ} \mathrm{C}}=4.24 \frac{\mathrm{V}}{{ }^{\circ} \mathrm{C}}
$$

From Figure 8,

$$
P_{u}=15.0 \mathrm{~s}
$$

Various PI settings are calculated from the above values of $K_{c_{u}}$ and $P_{u}$ using the formulas in Table 1. The PI settings are shown in Table 4. Both standard R$\mathrm{ZN}$ and enhanced R-ZN tuning are applied, with $k_{r}=1$ and $k_{r}=2$, respectively.

\subsubsection{Performance and stability robustness of the control system}

Figures 9, 10, 11, and 12 show responses in the air temperature $\left(y_{m f}\right)$ and the controller output $(u)$ due to a step change of the temperature setpoint $\left(y_{s p}\right)$ and a step change of the disturbance $(d)$ for the four different PI settings shown in Table 4. 

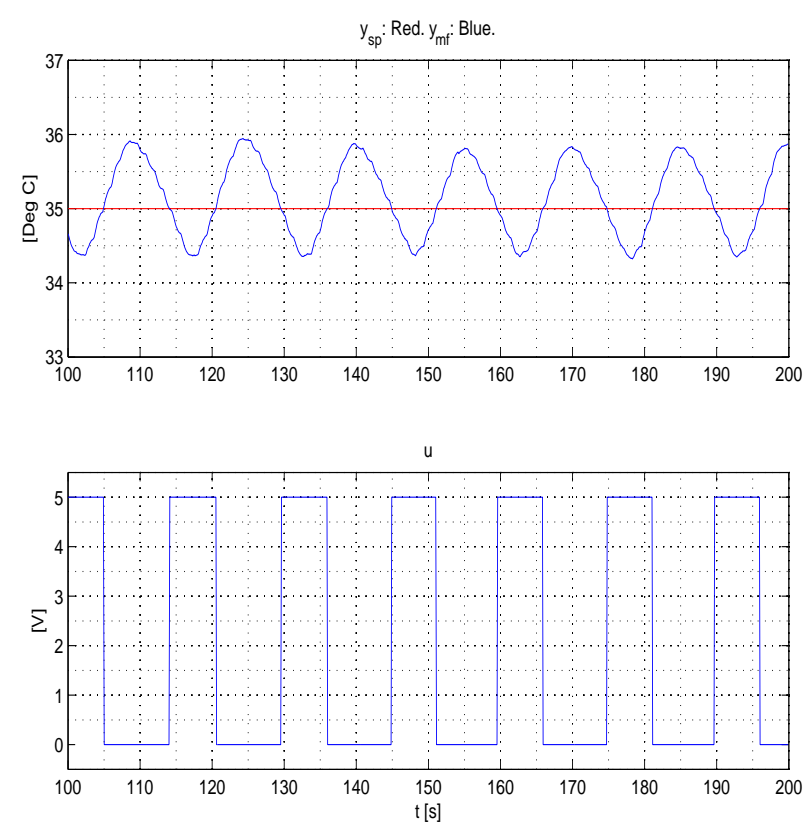

Figure 8: Responses during relay tuning

Performance and stability robustness measures are calculated from the time-series as explained in Section 3.2. $\mathrm{IAE}_{s}$ is calculated over the interval $t=[100 \mathrm{~s}, 180 \mathrm{~s}] . \quad \mathrm{IAE}_{d}$ is calculated over $t=$ $[200 \mathrm{~s}, 280 \mathrm{~s}]$. Table 4 summarizes the performance and robustness measures.

Table 4: Controller settings and performance and robustness measures for practical temperature control system for different PI settings.

\begin{tabular}{c|cccc} 
& $\mathbf{Z N}$ & $\begin{array}{c}\mathbf{R}-\mathbf{Z N} \\
k_{r}=1\end{array}$ & $\begin{array}{c}\mathbf{R - Z N} \\
k_{r}=2\end{array}$ & $\mathbf{T L}$ \\
\hline$K_{c}$ & 1.91 & 1.35 & 0.90 & 1.32 \\
$T_{i}[\mathrm{~s}]$ & 12.5 & 15.0 & 22.5 & 33.0 \\
$\mathrm{IAE}_{s}$ & 16.2 & 12.3 & 10.6 & 10.3 \\
$\mathrm{IAE}_{d}$ & 4.3 & 4.9 & 7.5 & 11.8 \\
$\mathrm{GM}$ & 1.5 & 1.8 & 2.8 & 2.6 \\
$\mathrm{GM}[\mathrm{dB}]$ & 3.5 & 5.1 & 8.9 & 8.3 \\
$\Delta \tau_{u}[\mathrm{~s}]$ & 1.6 & 2.6 & 7.7 & 5.7 \\
$P_{u}[\mathrm{~s}]$ & 24.0 & 31.0 & 50.0 & 39.0 \\
$\mathrm{PM}[\mathrm{deg}]$ & 24.0 & 30.2 & 55.4 & 52.6 \\
$T_{r}[\mathrm{~s}]$ & 3.8 & 4.9 & 8.0 & 6.2
\end{tabular}

Below are a number of observations made in Table 4 (the abbreviations are as in Table 4):

\section{- Setpoint tracking:}

$\mathrm{IAE}_{s}$ : TL and R-ZN with $k_{r}=1$ and with $k_{r}=2$ do not differ much and are clearly better than ZN which is due to the large overshoot and oscillatory response with $\mathrm{ZN}$.
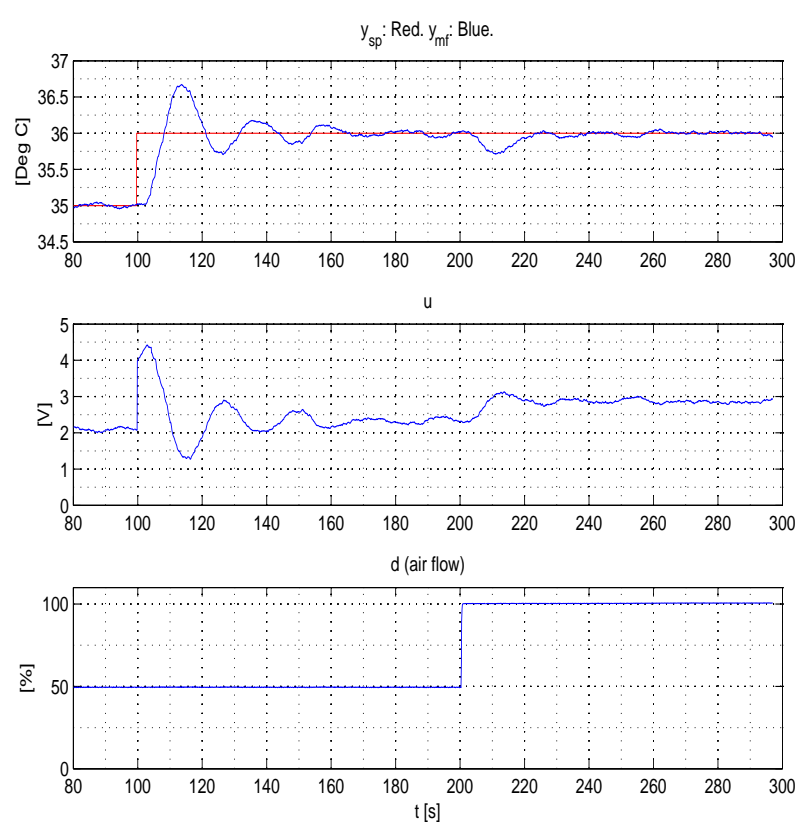

Figure 9: Responses with Ziegler-Nichols PI settings

$T_{r}$ : ZN is clearly best. It gives fast control. R-ZN with $k_{r}=1$ is also relatively fast.

- Disturbance compensation:

$\mathrm{IAE}_{d}: \mathrm{ZN}$ and R-ZN with $k_{r}=1$ are much better than both R-ZN with $k_{r}=2$ and TL. R-ZN with $k_{r}=1$ give only $36 \%$ of that of TL. Relaxed ZN with $k_{r}=2$ is also clearly better than TL.

- Stability robustness (margins):

GM: ZN is poor, and actually below the lower limit in ineq. (28). R-ZN with $k_{r}=1$ is small, but just within the limits.

PM: Again ZN is poor, and below the lower limit in ineq. (29). R-ZN with $k_{r}=1$ is small, but just within the limits. TL has a large value, possibly unnecessarily large since it is larger than the higher limit in ineq. (29). R-ZN with $k_{r}=2$ has a very large value.

The low stability margins with ZN are apparent in the oscillatory responses with the ZN settings. RZN with $k_{r}=1$ seems to give acceptable stability as seen from time-series. R-ZN with $k_{r}=2$ and TL both give smooth, but slow, responses.

\section{Comments and conclusions}

The Ziegler-Nichols PI settings give poor control loop stability. The TL and the R-ZN settings both with $k_{r}=1$ and $k_{r}=2$ give acceptable stability margins, though R-ZN with $k_{r}=1$ gives small margins. R-ZN 

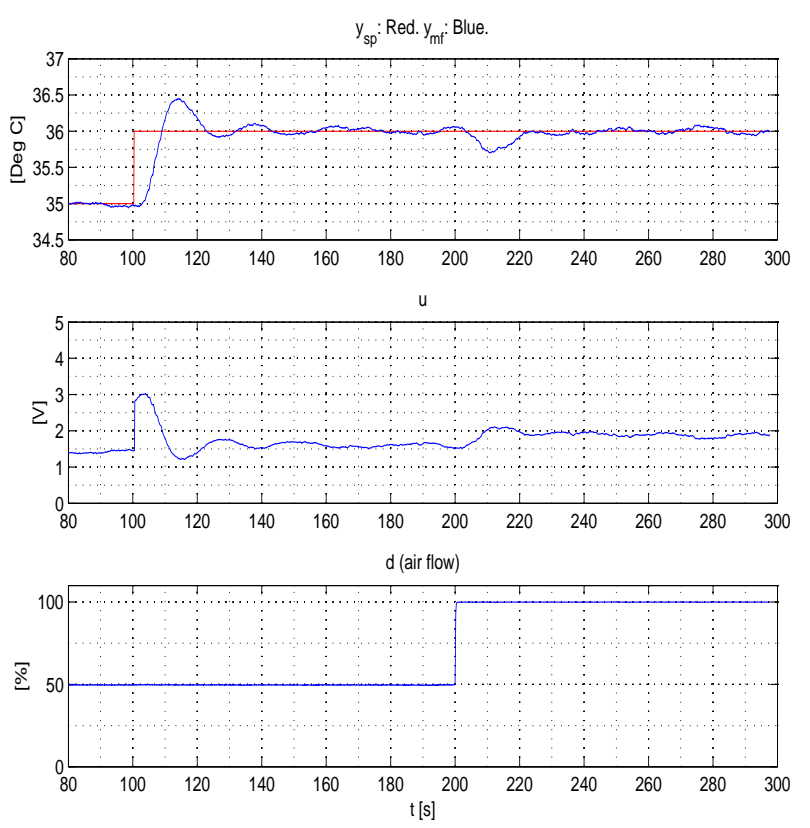

Figure 10: Responses with R-ZN PI settings with $k_{r}=$ 1.

with $k_{r}=1$ give clearly the best disturbance compensation, and since the stability margins are within the acceptable limits, it gives the prefered PI settings in this application.

If it is important with smooth responses, both TL and R-ZN with $k_{r}=2$ can be used. Among these two, we prefer the latter because it gives best disturbance compensation, and because the R-ZN settings are adjustable, while the TL settings are fixed.

\section{Relaxed tuning for processes with no time-delay but with lags}

Closed loop PI tuning with the standard ZieglerNichols method, the TL method, or even the R-ZN tuning method with the default setting $k_{r}=1$ may not work well if the process has no, or negligible timedelay, however, some lag is assumed. The resulting stability may be very poor. Such cases may occur in e.g. temperature control, Haugen et al. (2013) and biogas flow control of bioreactors, Haugen and Lie (2013). However, enhanced R-ZN tuning with a proper $k_{r}>1$ seems to work well. An explanation of the resulting poor stability is that, due to the lack of a time-delay, the phase characteristic is relatively flat around the critical frequencies, making the phase margin small.

Now, an extreme case is assumed, and enhanced RZN PI tuning is used. The value of $k_{r}$ that is found useful in this case may be used in other less extreme
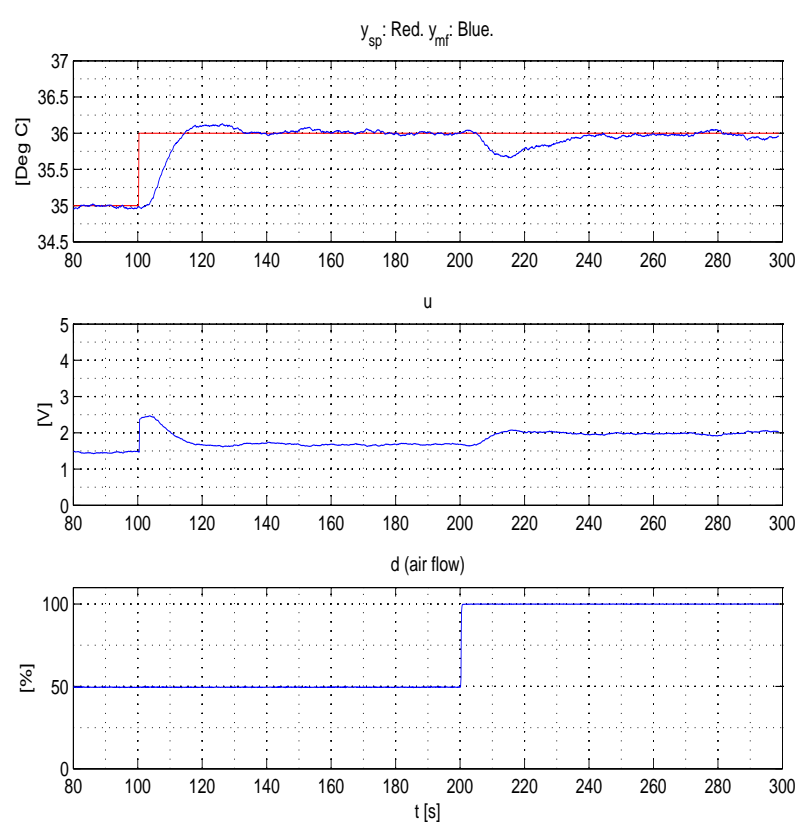

Figure 11: Responses with R-ZN PI settings with $k_{r}=$ 2.

cases to obtain proper stability. Note that for processes with a noteable time-delay the R-ZN PI settings with the default value $k_{r}=1$, i.e. eqs. (6)-(6), should be used.

Assume that the process is an integrator without any time-delay but with two lags in the form of timeconstant terms where one of the time-constants is one tenth of the other. Specifically, the following process transfer function model is assumed:

$$
y(s)=\frac{1}{s\left(T_{1} s+1\right)\left(T_{2} s+1\right)}\left[K_{i_{p}} u(s)+K_{d} d(s)\right]
$$

where $u$ is control variable and $d$ is disturbance. Timeconstant $T_{1}$ may represent a process lag due to e.g. dynamics of a heating element or a valve or a pump or represent inhomogeneous conditions in a tank, while $T_{2}$ may represent the time-constant of a measurement filter. The integrator, $1 / s$, may represent e.g. energy or material balance. The following parameter values are assumed: $K_{i_{p}}=1 \mathrm{~s}^{-1} K_{d}=1, T_{1}=1 \mathrm{~s}$, and $T_{2}=0.1 \mathrm{~s}$. In less extreme cases the difference between the two time-constants are less, and there may also be a non-zero time-delay.

The relay method is used, giving $K_{c_{u}}=10.24$ and $P_{u}=2.02 \mathrm{~s}$. The three PI tuning methods mentioned in the beginning of the present section are tested. Figure 13 shows their responses. With TL tuning and R-ZN tuning with $k_{r}=1$ the control system is stable, but the stability is poor. With Ziegler-Nichols tuning, the system is unstable! 

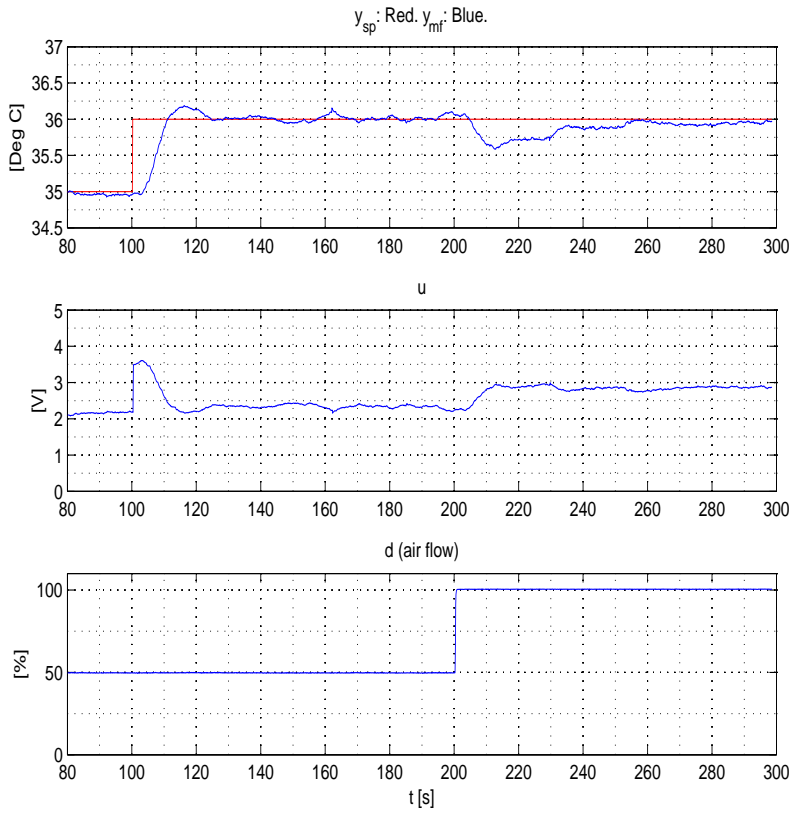

Figure 12: Responses with TL PI settings

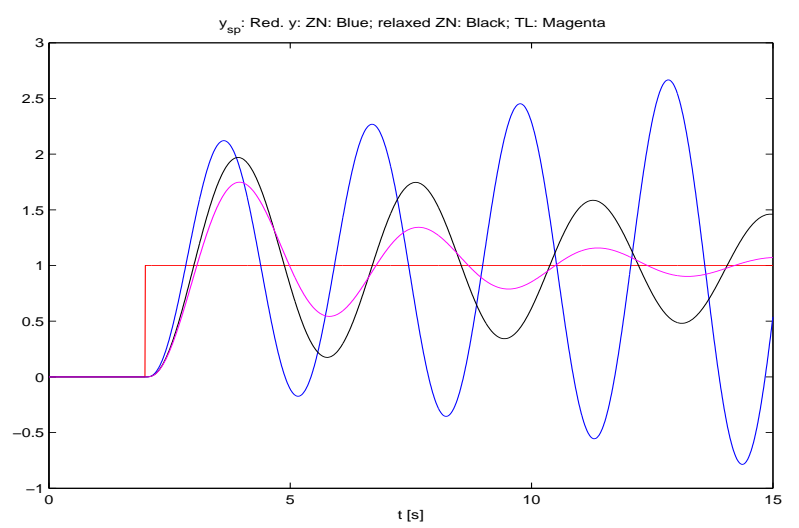

Figure 13: Responses with various PI settings.

By trial-and-error it is found that R-ZN tuning with $k_{r}=4$ works well. Hence, with $k_{r}=4$ in (18) and (19) the PI settings become

$$
K_{c}=0.13 K_{c_{u}}
$$

and

$$
T_{i}=2.5 P_{u}
$$

Figure 14 shows simulated responses. Table 5 shows PI settings and stability margins.

\section{Comments and conclusion:}

- GM is large, but is accepted here.

- PM is small and just outside the acceptable range where $30.0^{\circ}$ is the critical limit, cf. ineq. (29).
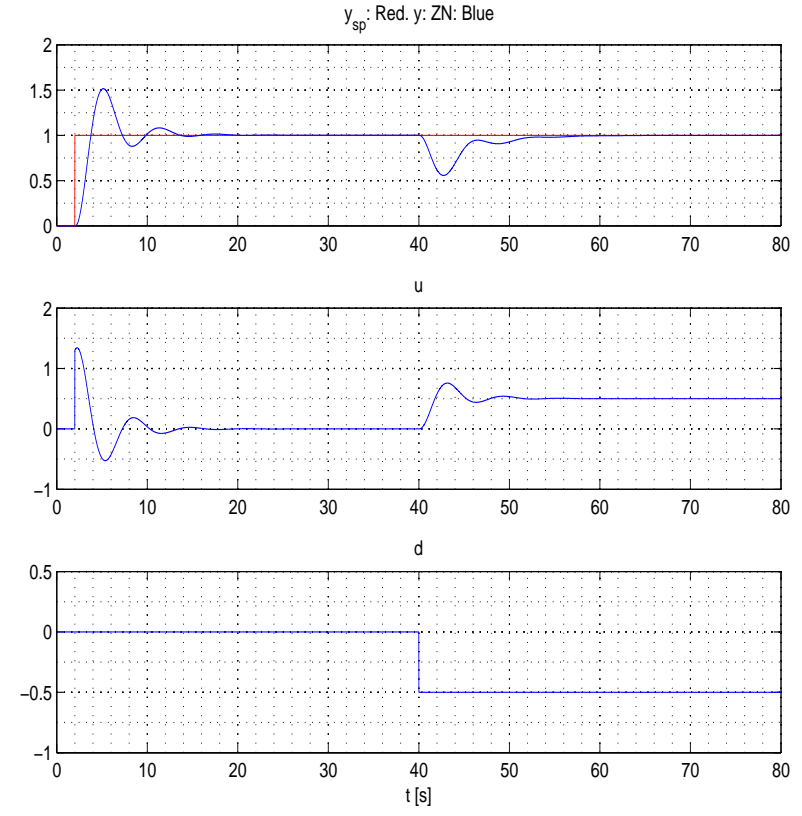

Figure 14: Responses with PI controller tuned with the R-ZN method with $k_{r}=4$.

Table 5: Controller settings and performance and robustness measures for simulated PI control system for an "integrator with two lags" process with R-ZN tuning with $k_{r}=4$.

\begin{tabular}{cc}
\hline$K_{c}$ & 1.3 \\
$T_{i}[\mathrm{~s}]$ & 5.2 \\
GM & 6.7 \\
GM [dB] & 16.5 \\
PM [deg] & 29.4 \\
\hline
\end{tabular}

However, the value of $29.4^{\circ}$ is here regarded as acceptable since it is for an assumed extreme case. With $k_{r}=5 \mathrm{PM}=34.3^{\circ}$ which is within the range given by ineq. (29), but simulations indicate that the control system becomes unnecessarily sluggish with $k_{r}=5$ applied for less extreme cases.

- How can one know that a process has one or more lags and no or negligible time-delay, so that the enhanced relaxed tuning should be applied? Physical insight may be useful: If the sensor or actuator is located close to the main process (which can be e.g. a reactor vessel), the time-delay may be negligible compared to time-constant lags. A process step response test is also an option, but then an open loop controller tuning method, as the Skogestad method (2003, 2004), may be applied directly. 


\section{Discussion}

The proposed new set of PI settings are based on tuning rules derived by Ziegler and Nichols (1942), tuning rules derived by Skogestad (2004), and the modeling of the process as an "integrator plus time-delay" according to $\mathrm{Yu}$ (1999). The validity and applicability of the proposed PI settings rely on assumptions made by these authors. The sensitivity of the present results with respect to such assumptions has not been investigated here. However, two simulation tests and one practical test indicate that the proposed tuning works as assumed.

In the simulations it is assumed that the process disturbance is an input disturbance as it acts on the process at the same place, dynamically, as the control signal does. In most practical processes the main disturbances are actually input disturbances. We have not investigated the consequences for our results of moving the disturbance to the process output.

It is found that for processes with no, or a negligible time-delay, but with some lags in the form of timeconstants, R-ZN tuning with $k_{r}=1$ may give poor stability (This applies to ordinary ZN and TL tuning, too). However, proper stability may be obtained with enhanced relaxation of the tuning, and $k_{r}=4$ seems to be a proper value at least for processes without timedelay but with two lags with one being one tenth of the other. The conditions that make the selection $k_{r}=4$ unsuccessful have not been investigated, but for processes where the time-constants are closer, the PI settings with $k_{r}=4$ will certainly be safe (conservative).

\section{Conclusions}

The main result of this paper is a proposed new set of PI settings which uses the same information as in the Ziegler-Nichols closed loop method, namely knowledge about the ultimate gain, $K_{c_{u}}$, and the ultimate period, $P_{u}$ : The proposed settings are:

$$
\begin{gathered}
K_{c}=0.32 K_{c_{u}} \\
T_{i}=P_{u}
\end{gathered}
$$

These settings are modifications, or relaxations, of the original Ziegler-Nichols PI settings, and they give improved control system stability. In this paper, the proposed setting have been successfully applied to two simulated control systems and to a practical temperature control system of an air heater.

Comparing with the TL PI settings, which also are based on knowledge of the ultimate gain and the ultimate period, the proposed PI settings give clearly better disturbance compensation.
The proposed PI settings have an adjustable parameter which can be used to obtain enhanced relaxation which is useful for processes with zero or negligible time-delay but some lags (time-constants).

\section{Acknowledgments}

Telemark University College has provided practical and economical support for the work with this paper. Thanks to colleague David Di Ruscio for useful discussions.

\section{A. Finding the ultimate gain and period from relay oscillations}

In the original Ziegler-Nichols closed loop method the user must find, typically by trial-and-error, the ultimate controller gain value, $K_{c_{u}}$, of a $\mathrm{P}$ controller which makes the responses in the control system become sustained oscillations. The user must also read off the ultimate period, $P_{u}$, of the oscillations. $K_{c_{u}}$ and $P_{u}$ are then used to calculate the PI settings with the following formulas:

$$
\begin{gathered}
K_{c}=0.45 K_{c_{u}} \\
T_{i}=\frac{P_{u}}{1.2}
\end{gathered}
$$

Åstrøm and Hägglund (1995) introduced a relay, or on-off, controller to replace the $\mathrm{P}$ controller in the tuning phase, thereby avoiding the possibly timeconsuming trial-and-error procedure as the oscillations come automatically. During the relay tuning the control signal is a square wave.

$K_{c_{u}}$ can be estimated from the relay oscillations as follows. Assume that the amplitude of the square wave is

$$
A_{\mathrm{sq}}=\frac{u_{\mathrm{on}}-u_{\mathrm{off}}}{2}
$$

where $u_{\text {on }}$ and $u_{\text {off }}$ are the values of the controller output when the relay is in the on- and off-state, respectively. The square wave is approximated by its fundamental sinusoidal component of its Fourier series. The fundamental sinusoid is known to have amplitude

$$
A_{\mathrm{sq}, \mathrm{F}}=\frac{4 A_{\mathrm{sq}}}{\pi}
$$

\section{Sinusoidal oscillations}

With relay-based oscillations, for many practical processes the filtered process measurement is approximately sinusoidal. Assume that the measurement has amplitude $A_{\text {sin }}$. The control error, which is the input 
to the relay, then also has amplitude $A_{\text {sin }}$. The equivalent gain of the relay function, which is used as the ultimate gain in eq. (41), is

$$
K_{c_{u}}=\frac{A_{\mathrm{sq}, \mathrm{F}}}{A_{\mathrm{sin}}}=\frac{4 A_{\mathrm{sq}}}{\pi A_{\mathrm{sin}}}=1.27 \frac{A_{\mathrm{sq}}}{A_{\mathrm{sin}}}
$$

The ultimate period, $P_{u}$, needed in eq. $(42)$ is the period of the oscillations.

\section{Triangular oscillations}

If the process dynamics is pure "integrator plus timedelay" the relay-based oscillations in the process measurement are not sinusoidal, but triangular. Let $A_{\text {tri }}$ be the amplitude of these triangular oscillations. The fundamental sinusoidal component of the triangular oscillation is known to have amplitude

$$
A_{\text {tri,F }}=\frac{8 A_{\text {tri }}}{\pi^{2}}
$$

The equivalent gain of the relay function, which is used as the ultimate gain in eq. (41), is

$$
K_{c_{u}}=\frac{A_{\mathrm{sq}, \mathrm{F}}}{A_{\mathrm{tri}, \mathrm{F}}}=\frac{\pi A_{\mathrm{sq}}}{2 A_{\mathrm{tri}}}=1.57 \frac{A_{\mathrm{sq}}}{A_{\mathrm{tri}}}
$$

The ultimate period, $P_{u}$, in eq. (42) is the period of the oscillations.

If the process dynamics is "time-constant plus timedelay" with the time-constant being much larger than the time-delay, and without other process dynamics (lags), the relay-based oscillations appear more triangular than sinusoidal. In these cases, eq. (47) can be used.

\section{B. Impact of the proposed parameter $c_{s}$ in the modified Skogestad PI settings}

Simulations are used to investigate the implications of using various values of parameter $c_{s}$ in eq. (9).

The process to be controlled is an "integrator with time-delay" process given by eqs. (30) with $K_{i_{p}}=1$ $\mathrm{s}^{-1}, K_{d}=1$ and $\tau=1 \mathrm{~s}$. The PI controller is tuned with the (modified) Skogestad tuning formulas, eqs. (8) and (9).

Figure 15 shows simulations for the following values of $c_{s}$ :

- $c_{s}=1.5$ which is the the value corresponding to the IMC settings for an "integrator with timedelay" process by Chien and Fruehauf (1990).

- $c_{s}=2$ which is the value used in the present paper.
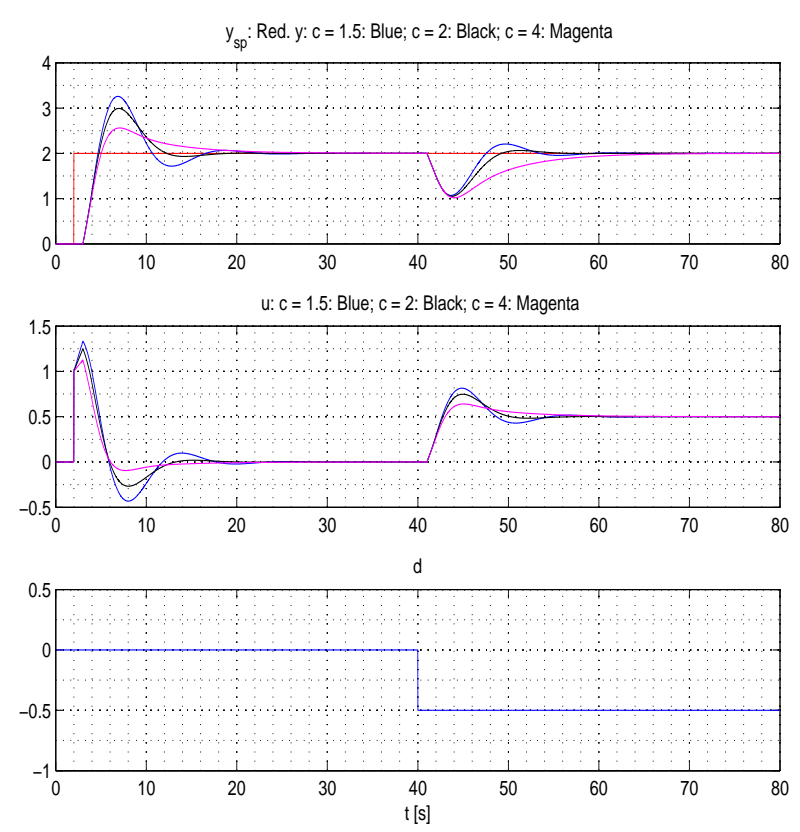

Figure 15: Simulations of control systems for an "integrator with time-delay" process with Skogestad controller tuning with $c_{s}=1.5, c_{s}=$ 2 , and $c_{s}=4$

- $c_{s}=4$ which is Skogestad's original value.

Table 6 shows a number of characteristics of the simulated control system for the three values of $c_{s}$. GM, $\mathrm{PM}$ and $T_{r}$ are calculated from the model, cf. Section 3.2. $\mathrm{IAE}_{s}$ is calculated time-series as explained in Section 3.2 over the interval $t=[2 \mathrm{~s}, 40 \mathrm{~s}] . \mathrm{IAE}_{d}$ is calculated over $t=[40 \mathrm{~s}, 80 \mathrm{~s}]$.

Table 6: Results with $c=1.5, c=2$, and $c=4$ in the (modified) Skogestad PI tuning formulas for an "integrator with time-delay" process.

\begin{tabular}{c|ccc} 
& $c_{s}=1.5$ & $c_{s}=2$ & $c_{s}=4$ \\
\hline$K_{c}$ & 0.5 & 0.5 & 0.5 \\
$T_{i}[s]$ & 3 & 4 & 8 \\
$\mathrm{IAE}_{s}$ & 9.6 & 8.1 & 7.8 \\
$\mathrm{IAE}_{d}$ & 4.5 & 4.5 & 8.0 \\
$\mathrm{GM}$ & 2.6 & 2.7 & 3.0 \\
$\mathrm{PM}[\mathrm{deg}]$ & 26.9 & 34.1 & 46.9 \\
$T_{r}[\mathrm{~s}]$ & 1.7 & 1.8 & 1.9
\end{tabular}

\section{Comments and conclusions}

In Table $6, \mathrm{PM}=26.9$ for $c_{s}=1.5$ which is regarded as a poor value since it is lower than the lower limit in ineq. (29). 
With $c_{s}=2$ and $c_{s}=4$ the stability margins are acceptable.

$\mathrm{IAE}_{d}$ with $c_{s}=2$ is $56 \%$ of $\mathrm{IAE}_{d}$ with $c_{s}=4$, indicating a considerable improved disturbance compensation with $c_{s}=2$. This is also clearly seen in the simulations.

We prefer $c_{s}=2$ over $c_{s}=4$ in the Skogestad PI settings formulas for "integrator plus time-delay" processes since the disturbance compensation is improved.

\section{Abbreviations and nomenclature}

\section{C.1. Abbreviations}

GM: Gain margin.

IAE: Integral of absolute error.

PI: Proportional plus integral (control).

PM: Phase margin.

R-ZN: Relaxed Ziegler-Nichols.

ZN: Ziegler-Nichols (original method).

TL: Tyreus-Luyben.

SIMC: Simple Internal Model Control.

\section{C.2. Nomenclature}

$A_{\text {sin }}$ : Amplitude of sinusoidal wave in control error or in process (output) measurement.

$A_{\mathrm{sq}}$ : Amplitude of square wave in control signal.

$A_{\text {tri }}$ : Amplitude of triangular wave in control error or in process (output) measurement.

$A_{u}$ : Amplitude of the on-off control signal.

$c_{s}$ : Parameter introduced in the integral time settings in the Skogestad method.

$d$ is process disturbance.

$\Delta$ : Deviation from operating point.

$e$ : Control error. $e=y_{\mathrm{sp}}-y$.

$k_{r}$ : The relaxation parameter in the Relaxed ZieglerNichols method.

$K$ is process gain.

$K_{c}[\mathrm{~s}]:$ Controller proportional gain.

$K_{d}$ is disturbance gain.

$K_{i_{p}}[\mathrm{~s}]$ : Process integrator gain.
$P_{u}[\mathrm{~s}]$ : Period of sustained oscillations.

$T[\mathrm{~s}]$ : Process time-constant.

$T_{c}[\mathrm{~s}]$ : Closed loop time-constant.

$T_{i}[\mathrm{~s}]$ : Controller integral time.

$T_{r}[\mathrm{~s}]:$ Response-time, or $63 \%$ rise time of step response.

$\tau[\mathrm{s}]:$ Process time-delay.

$u$ : Control signal (controller output).

$u_{\text {man }}$ : Manual control signal (control bias).

$y$ : Process output measurement.

$y_{\mathrm{sp}}:$ Setpoint

\section{References}

Åstrøm, K. J. and Hägglund, T. PID Controllers: Theory, Design and Tuning. ISA, 1995.

Chien, I. L. and Fruehauf, P. S. Consider IMC Tuning to Improve Controller Performance. Chem. Eng. Progress, 1990. Oct:33-41.

DiRuscio, D. On Tuning PI Controllers for Integrating Plus Time Delay Systems. Modeling, Identification and Control, 2010. 31(4):145-164. doi:10.4173/mic.2010.4.3.

Haugen, F. The good gain method for simple experimental tuning of pi controllers. Modeling, Identification and Control, 2012. 33(4):141-152. doi:10.4173/mic.2012.4.3.

Haugen, F. Air heater. http://home.hit.no/ finnh/ air_heater, 2013.

Haugen, F., Bakke, R., and Lie, B. Temperature control of a pilot anaerobic digestion reactor. Submitted to Modeling, Identification and Control, 2013.

Haugen, F. and Lie, B. On-off and pid control of methane gas production of a pilot anaerobic digestion reactor. Submitted to Modeling, Identification and Control, 2013.

Seborg, D. E., Edgar, T. F., and Mellichamp, D. A. Process Dynamics and Control. John Wiley and Sons, 2004. 
Skogestad, S. Simple analytic rules for model re- Yu, C. C. Autotuning of PID Controllers. Springer duction and pid controller tuning. Modeling, Verlag, 1999.

Identification and Control, 2004. 25(2):85-120. doi:10.4173/mic.2004.2.2.

Tyreus, B. D. and Luyben, W. L. Tuning PI Con-

Ziegler, J. and Nichols, N. Optimum settings for automatic controllers. Trans. ASME, 1942. 64(3):759trollers for Integrator/Dead Time Processes. Ind. Eng. Chem, 1992. 31(31). 